\title{
Gain-Scheduled Smith Predictor PID-based LPV Controller for Open-flow Canal Control
}

\author{
Y. Bolea, V. Puig and J. Blesa
}

\begin{abstract}
In this paper, a gain-scheduled Smith Predictor PID controller is proposed for the control of an open flow canal system that allows to deal with large variation in operating conditions. A linear parameter varying (LPV) control oriented model for open-flow channel systems based on a Second Order Delay Hayami (SODH) model is proposed. Exploiting the second order structure of this model, an LPV PID controller is designed using $\boldsymbol{H}_{\infty}$ and linear matrix inequalities (LMI) pole placement. The controller structure includes a Smith Predictor, real time estimated parameters from measurements (including the known part of the delay) that schedule the controller and predictor and unstructured dynamic uncertainty which covers the unknown portion of the delay. Finally, the proposed controller is validated in a case study based on a single real reach canal: the Lunax Gallery at Gascogne (France).
\end{abstract}

\section{INTRODUCTION}

$\mathrm{O}$ PEN flow canal systems have complex non-linear dynamics since they involve mass energy transport phenomena which behave as intrinsically distributed parameter systems and their complete dynamics is represented by partial differential hyperbolic equations (PDE) that are function of time as well as of spatial coordinates: The Saint-Venant's equations [1]. This system of non-linear partial differential equations has no known analytical solution in real geometry and it has to be numerically solved (characteristics method, Preissmann implicit scheme, etc.). The resulting simulation models are therefore suitable for scientific (time-consuming) simulations but are too complex for on-line control applications, as discussed in [1]. For this reason, in the literature more simplified linear time invariant (LTI) lumped parameter models (based on transfer functions) have been used to describe canals for control applications such as Hayami [2], Muskingum [3], Integrator Delay (ID) [4], Integrator Delay Zero (IDZ) [5] or black-box models identified using parameters estimation [6][7]. However, LTI lumped parameter models of open-flow canals are valid only around a given operating point since they are obtained through linearisation of Saint-Venant equations as already noticed and discussed in [1]. When considering large operating conditions, the coefficients and delay of the transfer function are not constant, but vary with the operating point (and/or different external factors) that determines the linearisation point. Consequently, the influence of the operating point on the parameters and delay should be taken into account in some way. Revising the literature, there are many works that consider LTI canal control models using different techniques, such as optimal control, adaptive control, predictive control, robust control, fractional control, etc. (see [3][8][9][10], among others) neglecting the effect of the parameter dependency with the operating point.

In this paper, the use of a linear parameter varying (LPV) model is proposed as a means to consider the parameters and delay variation with the operating point. This type of models relies on a linear lumped parameter model in which the parameters are not constant but a function of external parameters' and has been proposed by [11]. The idea of using LPV

\footnotetext{
This work has been funded by contract ref. HYFA DPI2008-01996 and WATMAN DPI2009-13744 of Spanish Ministry of Education.

The authors are with the Automatic Control Department and Institut de Robòtica i Informàtica Industrial, Universitat Politècnica de Catalunya and CSIC, Barcelona 08028, Spain (e-mail: \{yolanda.bolea,vicenc.puig,joaquim.blesa\}@upc.edu).

${ }^{1}$ In case that system varying parameters are function of system states and/or operating conditions the model is denoted as a quasi-LPV model .
} 
models to represent distributed parameter systems has already been proposed by [12]. Here, a quasi-LPV model of a reach canal suitable for control design based on a Second Order Delay Hayami (SODH) modelling approach is proposed. The LPV model is obtained by considering the dependence of the parameter models with the operating conditions given by the discharging flow. Preliminary results on the use of such a model for control have been presented in [13]. LPV models for canal control lead naturally to gain-scheduling (GS) techniques [11]. The benefits of using LPV GS techniques instead of robust control are obvious in terms of increase of performance in systems, such as canals, whose dynamics depends strongly on the operating point since model errors are partly due to neglecting such dependence [14].

In the literature, the main gain-scheduling control approaches are: conventional GS control [15], LPV GS control [16][17] and Takagi-Sugeno (TS) fuzzy GS control [18]. In this paper, LPV GS control is the considered approach. The use of TS GS approach is let as a further research. One of the main motivations of using LPV GS control instead of classical gain scheduling control is that the former as opposed to the latter guarantees stability and performance rigorously [19]. The LPV GS method guarantees closed loop stability based on the concept of quadratic stability (QS) for all real parameter trajectories inside a given region. Moreover, this methodology allows multi-objective criteria ( $H_{\infty}, H_{2}$, pole-placement) as well [16][17]. Under additional hypotheses, such kind of synthesis problems can be transformed into a convex optimisation problem involving linear matrix inequalities (LMIs). This results in a well behaved and computationally tractable problem. On the other hand, the heuristic gain scheduling controllers normally guarantee control system stability when the parameters are in a slow variation but sometimes may lead to instability or chaotic behaviour [19] when variations are faster. However, LPV GS control could not exploit the full dependence between the parameters and the operating point, since it would require solving an infinite number of LMIs, or instead solving a finite number of LMI using a grid of operating points but not guaranteeing stability rigorously. For this reason, in practice, the parameter region is bounded by a polytope what will allow to reduce the number of LMIs to be solved at its vertices [20].

In this paper, an LPV GS PID Smith Predictor controller (LPV PID+SP) is proposed as the control strategy to be used for controlling a single reach open flow canal. LPV GS design methodologies generally lead to high order controllers [16][17]. However, in practical applications, as the case of the control of open-canals, lower order controllers are preferred. In particular, PID controllers are widely used and accepted since most of the industrial controllers available in the market are based on a PID control law. For a general LPV system case, the design of a LPV PID controller should be formulated as an output feedback control that usually derives in solving a non-convex optimization problem based on Bilinear Matrix Inequalities (BMI) [20]. But, because of the second order structure of the Hayami model considered, a PID controller can be designed by reformulating it as a convex state-feedback problem that can be solved using pole placement based on LMIs and compensating the delay using a Smith Predictor. The controller is designed taking into account robust stability, performance, closed-loop pole constraints and essentially the time varying nature of the open-flow canal. The varying parameters are measured (estimated) in real-time and used to schedule PID parameters. A "delay scheduling" Smith Predictor scheme compensates most of the estimated delay. However, there is still a remaining delay due to the inaccuracy in its estimation that it will be represented as unstructured dynamic uncertainty in a robust control framework.

The structure of the paper is the following: In Section II, the Lunax gallery case study is described and the LPV Hayami modelling of the Lunax gallery is presented. In Section III, the formulation, synthesis and implementation of the PID control for a second order plus delay LPV system in LPV framework using a "delay scheduling" Smith predictor (Smith 
LPV PID) is introduced. In Section $I V$, the proposed methodology is assessed on the Lunax gallery case study and the control results are shown. In Section $V$, final conclusions are drawn.

\section{LUNAX GALLERY: SYSTEM DESCRIPTION AND LPV MODELLING}

\section{A. System Description}

The Lunax dam-gallery system is located at Gascogne, a SouthWestern region of France. This system is used to supply water to the river Gesse and consists of a single reach canal, a dam and a gate as depicted in Fig. 1a and 1b. The dam gate controls the upstream flow $\left(Q_{u p s}(t)\right)$ with a local controller (servo gate in Fig. 1c) in order to achieve an upstream reference flow $Q_{r}(t)$, that is $Q_{u p s}(t)=Q_{r}(t)$. The control objective aims at regulating the downstream flow $Q_{d n s}(t)$ at the output of the gallery for the full operating range (flow from $0.5 \mathrm{~m}^{3} / \mathrm{s}$ to $5 \mathrm{~m}^{3} / \mathrm{s}$ ). The downstream flow $Q_{d n s}(t)$ is measured indirectly through a limnimeter that provides the level $h_{d n s}(t)$ and by an experimental relation the flow, $Q_{d n s}(t)$ is determined.

The geometry of the gallery is circular, with diameter $d=1.8 \mathrm{~m}$, length $L=946.85 \mathrm{~m}$, slope $I=0.0026$ rad and the Manning coefficient $n=0.0154$.

The canal behaviour is modelled by Saint-Venant's equations [7] and reproduced using a high-fidelity simulator developed by the group of "Modelling and Control of Hydraulic Systems" at UPC [21]. This simulator solves numerically the SaintVenant's equations as usually is done by existent commercial open-flow canal simulators.

\section{B. Canal LPV modeling}

As discussed in the introduction, the Saint-Venant's equations are partial-differential equations for an arbitrary geometry lacks of analytical solution and are not useful for automatic control purposes. Here, as proposed by [14] in the context of control applications, the Saint-Venant equations can be approximated considering the two following assumptions that are also fulfilled in this case study: lateral discharges are null and inertia terms are negligible compared to one representing the energy lost by friction determined by the Hayami model that can be formulated in a LPV form (see [22] for more details) as

$$
G(s)=\frac{Q_{d n s}(s)}{Q_{u p s}(s)}=\frac{e^{-s \tau(\theta)}}{1+k_{1}(\theta) s+k_{2}(\theta) s^{2}}
$$

In this paper, the downstream flow has been chosen as scheduling variable, i.e, $\theta=Q_{d n s}$.

In order to estimate the variation of the LPV parameters $k_{1}(\theta), k_{2}(\theta)$ and $\tau(\theta)$ of Eq. (1) with the operating point, the multi-modelling algorithm presented in [23] has been used. This method divides the operating range in different regions: In the Lunax Gallery system (flow from $0.5 \mathrm{~m}^{3} / \mathrm{s}$ to $5 \mathrm{~m}^{3} / \mathrm{s}$ ) in four regions, bounded by lower and upper flows $\left[Q_{l o w_{i}}, Q_{u p_{i}}[\right.$. Then, a LTI model that describes the system in every region is determined. Once the LTI models have been identified, an LPV model can be obtained interpolating the values of the parameters with known functions (polynomials for example). 


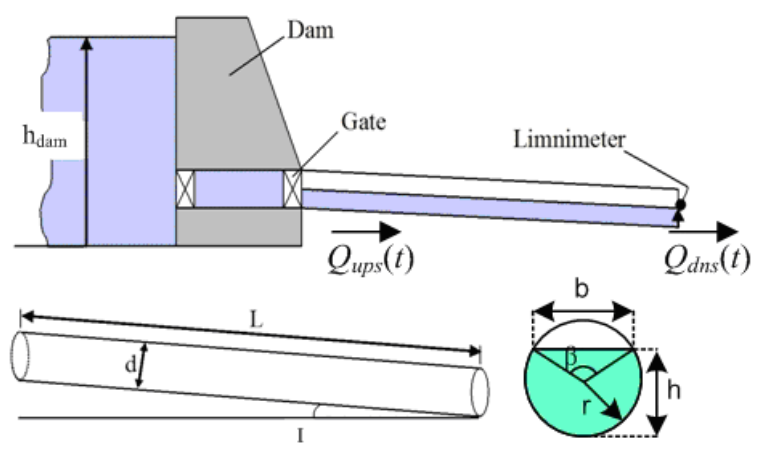

a)
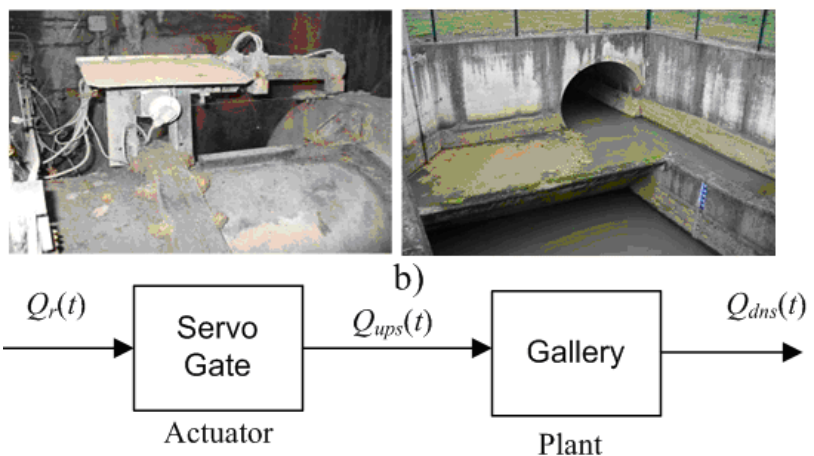

c)

Fig. 1. Lunax gallery. a) Geometrical scheme; b) Pictures; c) Functional scheme

TABLE I: PARAMETERS OF HAYAMI TRANSFER FUNCTION IN THE CONSIDERED OPERATING REGIONS

\begin{tabular}{ccccc}
\hline & {$\left[Q_{\text {lowi }_{i}}, Q_{u p_{i}}\left[\left(\mathrm{~m}^{3} / \mathrm{s}\right)\right.\right.$} & $k_{1}^{i}$ & $k_{2}^{i}$ & $\tau^{i}(\mathrm{~s})$ \\
$\mathrm{M}_{1}$ & {$[0.5,1[$} & 245 & 17360 & 252 \\
$\mathrm{M}_{2}$ & {$[1,2[$} & 234 & 15050 & 172 \\
$\mathrm{M}_{3}$ & {$[2,3.6[$} & 222 & 12310 & 110 \\
$\mathrm{M}_{4}$ & {$[3.6,5]$} & 199 & 8396 & 73
\end{tabular}

Here, the parameters of the LPV model have been obtained interpolating with a third order polynomial, the different model parameters at the central point of the operation region: $\mathrm{M}_{1}\left(Q_{1}^{c}=0.75 \mathrm{~m}^{3} / \mathrm{s}\right), \mathrm{M}_{2}\left(Q_{2}^{c}=1.5 \mathrm{~m}^{3} / \mathrm{s}\right), \mathrm{M}_{3}\left(Q_{3}^{c}=2.75 \mathrm{~m}^{3} / \mathrm{s}\right)$ and $\mathrm{M}_{4}\left(Q_{4}^{c}=4.25 \mathrm{~m}^{3} / \mathrm{s}\right)$. Then, the dependence of LPV model parameters (1) with respect to the scheduling variable $\theta$ is

$$
\begin{aligned}
& k_{i}(\theta)=k_{i 0}+k_{i 1} \theta+k_{i 2} \theta^{2}+k_{i 3} \theta^{3} \quad i=1,2 \\
& \tau(\theta)=\tau_{0}+\tau_{1} \theta+\tau_{2} \theta^{2}+\tau_{3} \theta^{3}
\end{aligned}
$$

The result of the parameter interpolation is summarized in Table II.

\begin{tabular}{ccccc}
\multicolumn{4}{c}{ TABLE II } & \multicolumn{4}{l}{ POLYNOMIAL COEFFICIENTS TRANSFER FUNCTION PARAMETERS } \\
\hline & 1 & $\theta$ & $\theta^{2}$ & $\theta^{3}$ \\
$k_{1}$ & 263 & -30.0 & 9.13 & -1.32 \\
$k_{2}$ & 2070 & -5324 & 1295 & -170 \\
$\tau$ & 363 & -178 & 39.7 & -3.24
\end{tabular}

The parameter variation ranges of the Hayami model (1) can be determined considering that the scheduling variable $\theta$ ranges in the interval $\left[0.5 \mathrm{~m}^{3} / \mathrm{s}, 5 \mathrm{~m}^{3} / \mathrm{s}\right]$ for the considered operating conditions. Thus: $k_{l}(\theta) \in[176,250], k_{2}(\theta) \in[5180$, $18300]$ and the time delay $\tau(\theta) \in[61 s, 284 s]$. Figs $2 \mathrm{a}$ and $2 \mathrm{~b}$ show the effect of parameter and delay variation in time and frequency responses. From these figures, it can be noticed that there is an important change in the time and frequency 
responses when the flow changes.
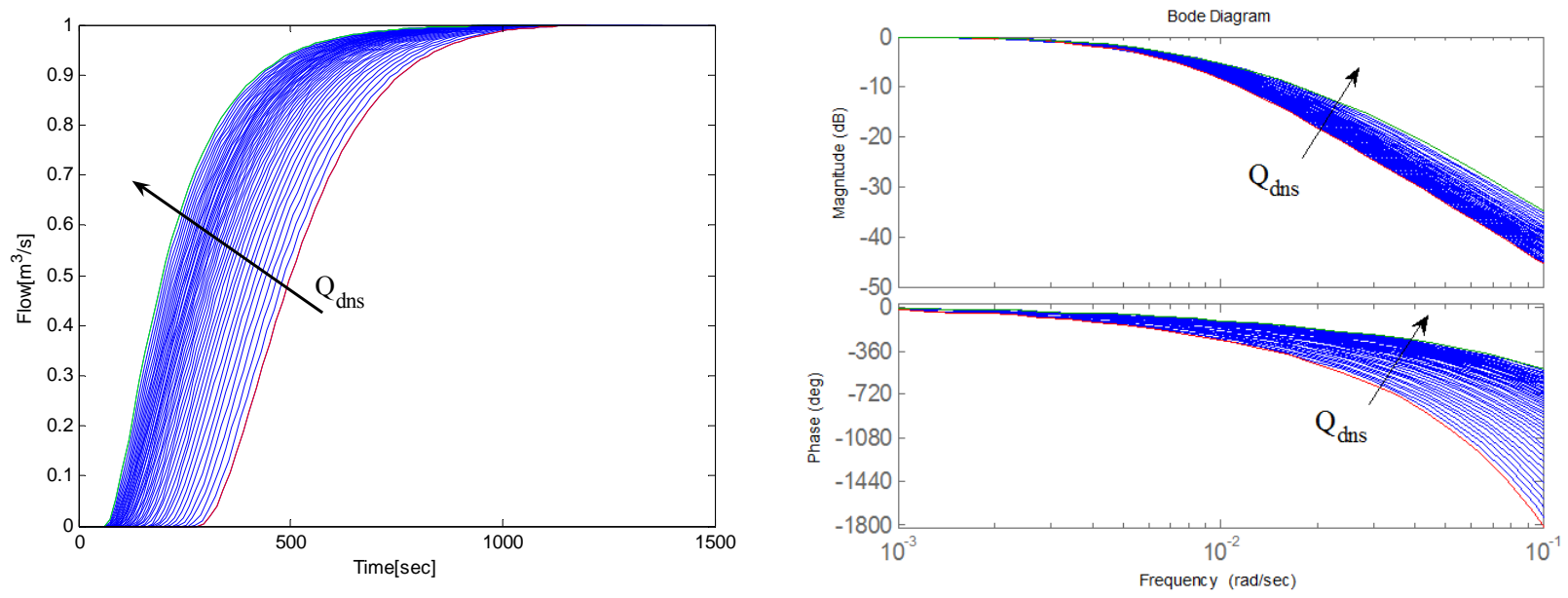

Fig. 2. (a) Step response and (b) Frequency response for different operating points

\section{Control structure}

For systems with significant transport delay (as the case of the Lunax Gallery), a controller including a Smith predictor (Figure 3a) allows to overcome the limitations of performance in case the controller alone is used [29]. Smith predictor allows the controller to be designed considering only the delay-free part of the plant since the delay is moved outside the loop as is shown in Figure $3 b^{2}$. For more detailed discussion see for example [29].

On the other hand, since the delay-free part of the plant (Lunax Gallery) is a second order system, the resulting natural controller obtained by the pole placement (or by the internal model control) method is a a PID (see [30][31]).

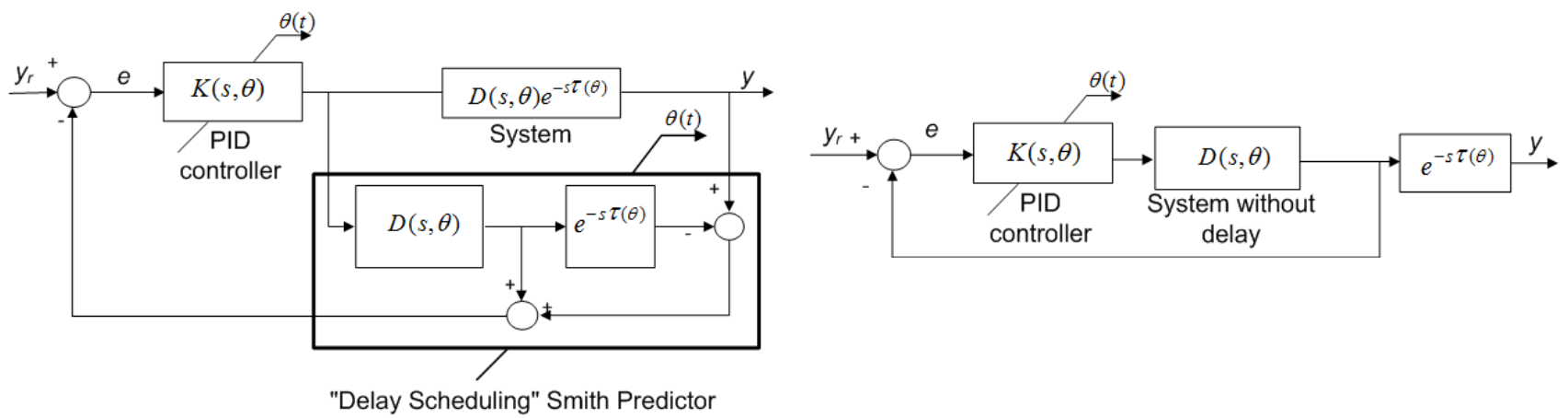

(a)

(b)

Fig. 3. (a) "Delay scheduled" Smith Predictor scheme. (b) Equivalent Smith Predictor closed-loop

In the open-flow canal literature, PI control has been usually used (see for example [1]). To see the potential benefit of using a PID controller in the Lunax Gallery case study, the tuning method for PID/PI based on a mixed sensitivity problem [28] is used. The design of PID/PI controllers using the Smith Predictor scheme will be done by considering the equivalent

${ }^{2}$ In case that there is no modeling uncertainty (i.e., there is a perfect match between the model and real system behaviors) and there are no disturbances. In case modeling uncertainty is present, it can be taken into account with the approach presented latter in this paper. 
feedback loop of Figure 3b, i.e., that allows the plant to be considered without delay. According to the method proposed in [28], there are significant benefits using a PID controller with respect to a PI when the parameter $\beta$ has a value in the range 5 to 10 . This parameter is defined as follows

$$
\beta=\frac{k_{\infty}}{k_{i} \tau}
$$

where:

$$
\begin{aligned}
k_{\infty} & =\frac{1}{D(0, \theta)} \min \left(3+\frac{2}{k_{150}}, 25\right) \\
k_{i} & =\frac{\omega_{150 G}}{D(0, \theta)}\left(\frac{0.45}{k_{150}+0.07}-0.1\right) \\
\tau & =\frac{1}{\omega_{150 G}\left(0.44+0.86 k_{150}\right)}
\end{aligned}
$$

In the previous expressions, $D(0, \theta)$ denotes the module of the plant frequency response at $\omega=0$ in the operating point $\theta$, i.e, $|D(j 0, \theta)|$. On the other hand,

$$
k_{150}=\frac{\left|D\left(j \omega_{150 G}, \theta\right)\right|}{D(0, \theta)}
$$

and $\omega_{150 G}$ is the frequency where the plant has a phase lag of $-150^{\circ}$ in the operating point $\theta$, i.e., $\arg D\left(j \omega_{150 G}, \theta\right)=150^{\circ}$.

The results obtained using the PI and PID controllers designed following [28] for different operating points are summarized in Table III. Figure 4 presents the frequency response of the open loop gain (controller in series with the plant without delay) plus the phase margins obtained with the PID and PI in these operating points. Notice from the results presented in this table that parameter $\beta$ obtained in the different operating point is in the range of 5 to 10 where significant benefits using a PID can be obtained. Notice only the case of the upper flow operating point $\left(\theta=4.25 \mathrm{~m}^{3} / \mathrm{s}\right)$ that leads to a value $\beta=4.8$ that although is not in the range of 5 to 10 is very close to the lower limit of this range. In fact according to [28], the index that measures the quality of control $\left(J_{v}=\left\|\frac{1}{s} \frac{D(s, \theta)}{1+K(s, \theta) D(s, \theta)}\right\|_{\infty}\right)$ is smaller in the case of a PID than in the case of a PI, at the price of not increasing so much the control effort $\left(J_{u}=\left\|\frac{K(s, \theta)}{1+K(s, \theta) D(s, \theta)}\right\|_{\infty}\right)$, and preserving a better phase margin. All these can be seen in detail in Table III. For this reason, for the considered case study (Lunax Gallery), a PID instead of a PI will be used in this paper. 
Table III. PID and PI designed using tuning method proposed by Kristiansson and Lennartson [28]

\begin{tabular}{|c|c|c|c|c|c|c|c|}
\hline \multicolumn{1}{c|}{$\begin{array}{c}\text { Operating } \\
\text { Point }\end{array}$} & $\beta$ & \multirow{2}{*}{$\begin{array}{c}\text { PID Phase } \\
\text { Margin }\end{array}$} & \multirow{2}{*}{$\begin{array}{c}\text { PI Phase } \\
\text { Margin }\end{array}$} & \multicolumn{2}{|c|}{ PID } & \multicolumn{3}{c|}{ PI } \\
\hline$\theta=0.75 \mathrm{~m}^{3} / \mathrm{s}$ & 5.9 & 48.7 & 33.6 & 25 & 12.2 & 9.3 & 45.6 \\
\hline$\theta=1.5 \mathrm{~m}^{3} / \mathrm{s}$ & 5.8 & 48.8 & 34.2 & 25 & 11.1 & 9.45 & 41.8 \\
\hline$\theta=2.75 \mathrm{~m}^{3} / \mathrm{s}$ & 5.53 & 47.6 & 34.6 & 25 & 9.8 & 10.3 & 34.7 \\
\hline$\theta=4.25 \mathrm{~m}^{3} / \mathrm{s}$ & 4.8 & 46.2 & 35.6 & 25 & 7.2 & 11.7 & 25.2 \\
\hline
\end{tabular}
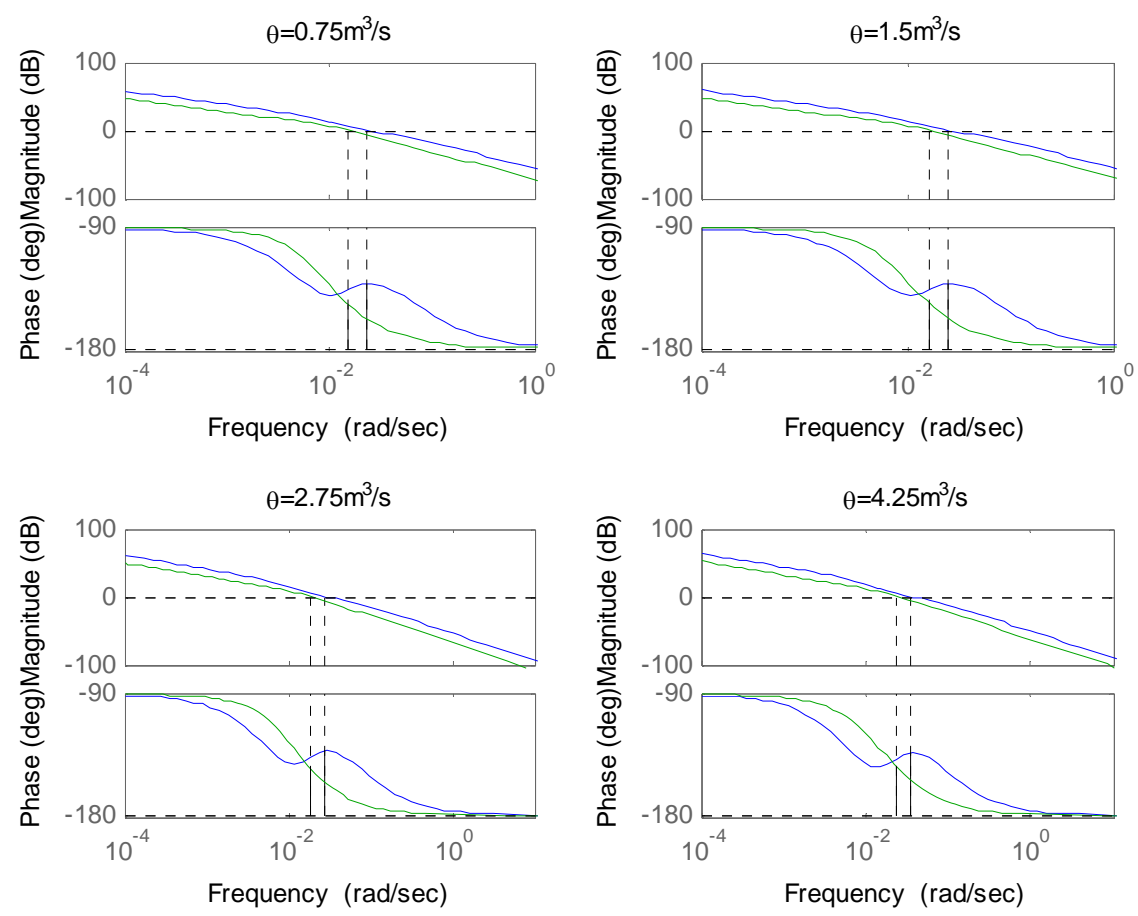

Fig. 4. Frequency response (and phase margins) of the PID (blue) and PI (green) in series with the delay-free part of the plant in the considered operating points

\section{GAIN SCHEDULING SMITH LPV PID CONTROL DESGIN METHODOLOGY}

\section{A. Problem set-up}

As discussed in the introduction, the objective of this paper is to design a gain-scheduling controller using LPV theory for the plant model described by (1). This controller will be able to adapt its parameters to the variations in the plant dynamics in order to maintain stability and high performance along all trajectories $\theta(t)$. In other words, the controller is 'selfscheduled', that is automatically gain-scheduled with respect to $\theta(t)$. The controller structure will combine a PID law with a Smith Predictor as discussed in Section II.

The variable delay in the Hayami model (1) can be handled with either of the two different ways:

1) As an LTI dynamic uncertainty covered conveniently by a weight $W_{\Delta}$ as in [24]. 
2) As a time-varying parameter which updates a Smith Predictor.

The first approach could be conservative, and unnecessarily decrease the overall performance. On the other hand, the second approach could provide a far better performance, but it does not take into account the measurement error of the time-varying delay $\tau(\theta)$. In this paper, it is proposed to combine both approaches by assuming that a real time estimation $\hat{\tau}(\theta)$ of the delay is available by means of (2), which will be used to update the Smith Predictor (Fig. 3a). The difference between the actual and the estimated delay is considered as global dynamic uncertainty as in [24][25] and is used in the design and robustness conditions. Therefore, we assume that the time delay dynamics has a time varying nature although its estimation error dynamics is time invariant, with a constant bound. Proceeding in such a way, most of the delay is compensated and the remaining portion, denoted as

$$
\Delta \tau(\theta)=\tau(\theta)-\hat{\tau}(\theta)
$$

can be covered by LTI unstructured uncertainty. This measurement error is always smaller than the actual delay, therefore the uncertainty is less conservative, which in turn has a lower impact on performance. This uncertainty is handled here as multiplicative output uncertainty and is bounded through the following uncertainty bound (weight) that "covers" the delay measurement error frequency response as tightly as possible following [24][25]:

$$
W_{\Delta}(s, \Delta \tau)=\frac{2.05 \Delta \tau_{\max } s}{\Delta \tau_{\max } s+1} \quad \text { with } \quad \Delta \tau(\theta) \leq \Delta \tau_{\max }
$$

Although the delay is time varying, by assuming that the delay measurement error is time invariant, the same robust stability analysis of the Smith Predictor proposed in [25] for the LTI case can be performed. This is due to the fact that the remaining system, after the cancellation of delay with the use of its estimation, can be considered as finite dimensional LTI, according to this assumption. Therefore, the delay scheduled Smith Predictor eliminates the infinite dimensional as well as the time varying nature of the delay, reducing it to a LTI dynamic uncertainty.

\section{B. Design procedure}

For a general LPV system case, the design of a LPV PID controller should be formulated as an output feedback control that usually derives in solving a non-convex optimisation problem based on BMI [20]. However, considering the second order structure of model (1), a convex state feedback problem can be formulated (see [26] for details). In more detail, rewriting the LPV model (1) of the Lunax gallery as follows

$$
G(s, \theta)=\frac{b_{0}(\theta)}{s^{2}+a_{1}(\theta) s+a_{0}(\theta)} e^{-s \tau(\theta)}
$$

where: $a_{1}(\theta)=k_{1}(\theta) / k_{2}(\theta)$ and $a_{0}(\theta)=b_{0}(\theta)=1 / k_{2}(\theta)$, the following state space description can be obtained:

$$
\begin{aligned}
\dot{x} & =A(\theta) x+B(\theta) u+B_{r} r, \\
u & =-K(\theta) x+K_{P}(\theta) r+K_{D}(\theta) \dot{r}, \\
y & =C x,
\end{aligned}
$$


where $y$ is the system output, $x=\left[\begin{array}{lll}x_{1} & x_{2} & x_{3}\end{array}\right]^{T}$ is the state with variables defined by $x_{1}=y, x_{2}=\dot{x}_{1}$, $x_{3}=-\int e d t, e=r-y, r$ the reference input, and

$$
A(\theta)=\left[\begin{array}{ccc}
0 & 1 & 0 \\
-a_{0}(\theta) & -a_{1}(\theta) & 0 \\
1 & 0 & 0
\end{array}\right], B(\theta)=\left[\begin{array}{c}
0 \\
b_{0}(\theta) \\
0
\end{array}\right], B_{r}(\theta)=\left[\begin{array}{c}
0 \\
0 \\
-1
\end{array}\right] C=\left[\begin{array}{lll}
1 & 0 & 0
\end{array}\right], K(\theta)=\left[\begin{array}{lll}
K_{P}(\theta) & K_{D}(\theta) & K_{I}(\theta)
\end{array}\right] .
$$

Using this state-space model, the LPV PID controller design for the system described by (5) will now be formulated as a state feedback problem and embedded in a self-scheduled LPV control problem as developed by [16][17], briefly summarised in this section. The control design specifications that will be considered are a mixture of performance and robustness objectives arranged as a mixed sensitivity problem (MSP) [24] (see Fig. 5), as follows:

$$
\left\|\left[\begin{array}{lll}
W_{e} S & W_{u} K S & W_{\Delta} T
\end{array}\right]\right\|_{\infty}^{T}<\gamma \leq 1
$$

Here $S$ is the sensitivity and $T$ is the complementary sensitivity functions. These transfer functions represent weighted tracking error (or disturbance rejection), weighted control action and robust stability, respectively. The weight for the complementary sensitivity, $W_{\Delta}$, captures the uncertainty of the plant model (in this case coming from the delay measurement error). The weights $W_{e}$ and $W_{u}$ allows to find a compromise between performance and control effort, respectively. Robustness is presented as an $H_{\infty}$ bound and is related with the dynamic uncertainty coming from the real time delay estimation error. Performance is a combination of weighted error and control action minimization measured in terms of the energy integrals of the input and output signals involved. A PID controller is a good approximation of a robust high order controller at low frequencies, especially because of the inclusion of the integral action. Then, the resulting PID controller is expected to preserve the disturbance rejection performance of a high-order controller. Furthermore, the time response is tuned via a selected closed loop pole placement LMI region [27]. This control design problem will be solved using the notion of QS and closed loop pole placement applied to a MSP, considering the delay measurement error as multiplicative dynamic uncertainty (see Section III.A).

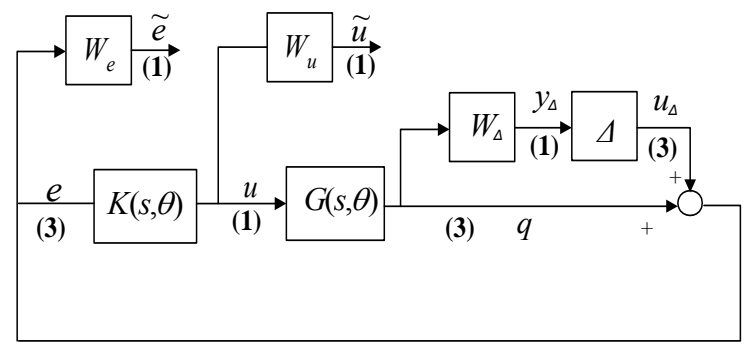

Fig. 5. MSP scheme

To achieve a PID controller as a state feedback when solving the MSP problem (8), it is necessary to additionally consider the following issues so that the order of the augmented model does not increase:

1) The performance and control effort weight functions need to be constants $\left(W_{e}=D_{e}, W_{u}=D_{u}\right)^{3}$,

3 According to [24], performance and control effort weight functions can be defined as follows: $W_{e}=\left(s / M_{s}+\omega_{b}\right) /\left(s+\omega_{b}\right)$ and 
2) The uncertainty weight in (4) is modified as follows:

$$
\widetilde{W}_{\Delta}(s, \Delta \tau)=2.05 \Delta \tau_{\max } s
$$

where that $W_{\Delta}=\widetilde{W}_{\Delta} G_{f}$.

Under the previous considerations, using a Smith Predictor scheme (Fig. 3a) and the uncertainty weight introduced in (9) bounding the delay measurement error (3), the following system representation is achieved (Fig. 5) [16][17]:

$$
\begin{aligned}
& \dot{x}(t)=A(\theta) x(t)+B(\theta) u(t)+B_{u_{\Delta}}(\theta) u_{\Delta}(t) \\
& z(t)=C_{z}(\theta) x(t)+D_{z u}(\theta) u(t)+D_{z u_{\Delta}}(\theta) u_{\Delta}(t) \\
& q(t)=C_{q}(\theta) x(t)+D_{q u}(\theta) u(t)+D_{q u_{\Delta}}(\theta) u_{\Delta}(t)
\end{aligned}
$$

with

$$
\begin{aligned}
& x=\left[\begin{array}{lll}
x_{1} & x_{2} & x_{3}
\end{array}\right]^{T}=\left[\begin{array}{lll}
y & x_{\Delta} & x_{I}
\end{array}\right]^{T}=\left[\begin{array}{lll}
y & \dot{x} & x_{I}
\end{array}\right]^{T}, \underline{u}=\left[\begin{array}{ll}
u_{\Delta} & u
\end{array}\right]^{T} z=\left[\begin{array}{lll}
y_{\Delta} & \tilde{u} & \tilde{e}
\end{array}\right]^{T}, A(\theta)=\left[\begin{array}{ccc}
0 & 1 & 0 \\
-a_{0}(\theta) & -a_{1}(\theta) & 0 \\
1 & 0 & 0
\end{array}\right], \\
& \left.B(\theta)=\left[\begin{array}{ll}
0 & b_{0}(\theta)
\end{array}\right]\right]^{T}, B_{u_{\Delta}}(\theta)=\left[\begin{array}{lll}
0 & 0 & 0
\end{array}\right]^{T}, C_{z}(\theta)=\left[\begin{array}{ccc}
D_{\Delta} & C_{\Delta} & 0 \\
0 & 0 & 0 \\
-D_{e} & 0 & 0
\end{array}\right], C_{q}(\theta)=\left[\begin{array}{lll}
-1 & 0 & 0
\end{array}\right], D_{z u}(\theta)=\left[\begin{array}{lll}
0 & D_{u} & 0
\end{array}\right]^{T}, \\
& D_{z u_{\Delta}}=\left[\begin{array}{lll}
0 & 0 & -D_{e}
\end{array}\right]^{T}, D_{q u_{\Delta}}=-1, D_{q u}=0 \text {. }
\end{aligned}
$$

Once the MSP problem (8) is formulated as a linear fraction transformation (LFT), is solved recasting the following theoretical results concerning:

1) Quadratic $H_{\infty}$ performance [16].

2) Robust and Quadratic D-Stability [17][27].

\section{Implementation details}

Since the parameter $b_{0}(\theta)$ of the system in (5) varies with parameter $\theta$, to fulfil hypothesis associated to the self-scheduled $H_{\infty}$ control synthesis problem for LPV systems developed by [16][17], the following procedure can be used. First, the LPV gain-scheduling PID controller $K(\mathrm{~s}, \theta)=K\left[\mathrm{~s}, a_{0}(\theta), a_{I}(\theta)\right]$ is designed taking into account only the variation of the parameters $a_{0}(\theta)$ and $a_{1}(\theta)$, and assuming that the parameter $b_{0}(\theta)$ has a nominal value $b_{0 n o m}$. Finally, keeping the same inner loop through equation

$$
\tilde{K}\left[s, a_{l}(\theta), a_{0}(\theta), b_{0}(\theta)\right]=K\left[s, a_{l}(\theta), a_{0}(\theta)\right] \frac{b_{0}(\theta)}{b_{0 \text { nom }}}
$$

the variation of parameter $b_{0}(\theta)$ is considered in the design of the controller. Due to the fact that the time varying

$W_{u}=\left(s+\omega_{b c} / M_{u}\right) / \omega_{b c}$ where $M_{s}=\|S\|_{\infty},|S(0)| \leq \varepsilon$ and $|K S(0)| \leq M_{u}$. In case these weights should be selected to be constant, they can be defined as $W_{e}=1 / \varepsilon$ and $W_{u}=1 / M_{u}$. 
parameters $a_{0}(\theta)$ and $a_{1}(\theta)$ enter affinely in the augmented model equations (see (10) and (11)), the parameter region is approximated by a box $\Theta$ defined by the parameter variation ranges of the parameter $\left(\left[a_{0 \min }, a_{0 \max }\right]\right.$ and $\left.\left[a_{1 \min }, a_{1 \max }\right]\right)$ thanks to the transformation introduced by (12), the model of the LPV system can be represented by:

$$
\left[\begin{array}{ll}
A(\theta) & B(\theta) \\
C(\theta) & D(\theta)
\end{array}\right]=\sum_{i=1}^{r} \lambda_{i}\left[\begin{array}{cc}
A_{i} & B_{i} \\
C_{i} & D_{i}
\end{array}\right]
$$

The delay $\tau(\theta)$ has already been considered as a scheduled (time varying) parameter in the Smith Predictor implementation, and the delay estimation error bounded by a multiplicative uncertainty in the design process, as explained in Section III.A and III.B. The resulting static time varying state feedback controller obtained after the solution of (8) can be transformed by the equivalence introduced in Section III.B, in a PID controller as in (7). This controller schedules the parameters $\left(a_{0}(\theta), a_{1}(\theta)\right)$ and by means of the transformation in (12), the scheduling of parameter $b_{0}(\theta)$ is added. This controller guarantees QS and Quadratic $H_{\infty}$ Performance, as well as ("frozen") closed loop pole location inside the desired LMI region. Since the plant is polytopic, the controller $K(s, \theta)=K(\theta)$ is designed as well in a polytopic way and implemented according to:

$$
K(\theta) \in \operatorname{Co}\left\{K\left(v_{1}\right), K\left(v_{2}\right), \ldots, K\left(v_{r}\right)\right\}:=\left\{\sum_{i=1}^{r} \lambda_{i} K_{i} ; K_{i}=K\left(v_{i}\right)\right\}
$$

where: $\sum_{i=1}^{r} \lambda_{i}(\theta)=1, \lambda_{i}(\theta)>0$.

This technique is known as a convex decomposition technique, and $C o$ is the function that generates the convex hull of the polytope vertices. The polytopic coordinates are calculated in such a way that each vertex $v_{i}, i=1, \ldots, r$ has coordinates:

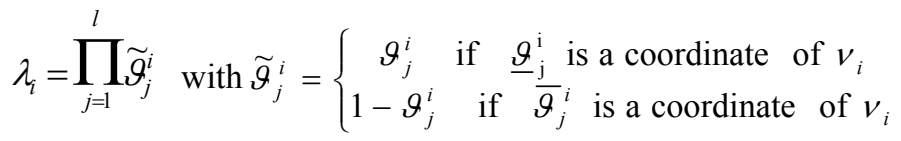

where $\widetilde{\vartheta}_{j}^{i}=\frac{\left(\bar{\theta}_{j}^{i}-\theta_{j}^{i}\right)}{\left(\bar{\theta}_{j}^{i}-\underline{\theta}_{j}^{i}\right)}, j=1, \ldots, l$, and $\left(\underline{\theta}_{j}^{i}, \bar{\theta}_{j}^{i}\right)$ represent the upper and lower bounds of $\theta_{j}^{i}$, and $l$ fulfils that $r=2^{l}$.

\section{TEST CASE RESULTS}

In the Lunax gallery case study, presented in Section II, the downstream flow $Q_{d n s}$ will be controlled using the proposed gain scheduling LPV PID control methodology described in Section III. The PID controller will be gain scheduled using as scheduling variable $\theta=Q_{d n s}$. The control objective in the Lunax gallery is that the downstream flow $Q_{d n s}$ follows a reference flow $Q_{r}$ with the pre-specified control specifications regarding the error and closed loop response using a control signal value in the range of the actuator operation.

\section{A. Controller design ${ }^{4}$}

The LPV parameters of the Hayami transfer function can be determined in terms of the scheduling variable $\theta$, as shown in Section II. For control design purposes, the model is expressed as in (5) and the scheduling variable varies in the 
range $\theta \in[0.5,5]$. Additionally, the delay estimation error is bounded by $\Delta \tau(\theta) \in[0.1,5] \mathrm{s} \mathrm{comparing}$ the polynomial that is used to estimate the delay introduced in (2) with the real delay measured from the canal response. The error in the time delay is taken into account in the control design as an LTI unstructured multiplicative uncertainty bounded by a uncertainty weight $\left(W_{\Delta}(s)=10.25 s /(5 s+1)\right)$ and approximated by $W_{\Delta}(s, \Delta \tau)=10.25 s$. Once the main time varying delay has been compensated by the Smith Predictor (see Fig. 3a) and the remaining delay error considered as the weight $W_{\Delta}(s, \Delta \tau)$ of a multiplicative dynamic uncertainty (see Section III.A and III.B), a PID controller is designed as a state feedback. This controller should guarantee closed loop stability and the following (step response) performance specifications:

- minimisation of the tracking error,

- control signal within $[0.5,5]$ and

- closed-loop damping of $\xi \geq 0.4$ and settling time in $t_{s s} \approx 230$ (minutes),

for any arbitrarily fast parameter variation. According to (Skogestad et al., 1997), the tracking error and the bounded control signal specifications can be achieved by the appropriate selection of the weights $\left(W_{e}\right.$ and $\left.W_{u}\right)$. As discussed in Section III, in order not to increase the order of the controller the previous weights are selected according to (Skogestad et al., 1997) but constant as follows: $W_{e}=1$ and $W_{u}=1 / 5=0.2$. Finally, to achieve the desired closed-loop behaviour, LMI pole placement is used. To this end, a LMI region $S\left(h_{1}, h_{2}, \alpha\right)$ is defined as a combination of three subregions:

- A conic sector with apex at $x=0$ and angle $\alpha=3 \pi / 4$, which captures the closed-loop damping constraint $\xi \geq 0.4$.

- Left half plane that guarantees the maximum settling time $\left(h_{1}=-0.016\right)$.

- Left half plane that guarantees the minimum settling time $\left(h_{2}=-0.0018\right)$.

Once the MSP problem with pole placement constraints problem (8) is formulated, the gains of LPV PID controller are obtained by solving LMI's obtained from Quadratic $H_{\infty}$ Performance [16] and Quadratic D-stability [17][27] at each vertex of the interval box of $a_{1}(\theta)$ and $a_{0}(\theta)$ described by $a_{1}(\theta) \in[0.009617,0.04826]$ and $a_{0}(\theta) \in[5.4600 e-5,1.1931 e-4]$, as indicated in [16]. Table IV shows the values of the LPV PID controller obtained in the different vertices.

\begin{tabular}{cccc} 
TABLE IV: VALUES OF THE LPV PID CONTROLLER OBTAINED IN THE DIFFERENT VERTICES \\
\cline { 1 - 4 } Vertex & $K_{P}$ & $K_{I}$ & $K_{D}$ \\
1 & 16.4500 & 0.2120 & 295.8225 \\
2 & 38.6900 & 0.4987 & 388.2844 \\
3 & 18.2013 & 0.2412 & 336.5728 \\
4 & 24.5335 & 0.3252 & 341.2868
\end{tabular}

\section{B. Results}

For comparison purposes, a robust LTI $H_{\infty}$ PID controller with a standard LTI Smith Predictor is also designed. The LTI model used corresponds to the model obtained in the lower flow regime (i.e., for $0.5 \mathrm{~m}^{3} / \mathrm{s}$ ) that corresponds to the case that the transport delay is longer. Parameter and delay variations are considered as uncertainty, while the control objectives are the same as in the case of the LPV PID with SP. The detailed design procedure used can be found in [27]. 
Fig. $6 \mathrm{a}$ and $6 \mathrm{~b}$ present the comparisons of the closed loop response when both LTI $H_{\infty}$ PID and the proposed LPV PID controllers are used in different operating points. Fig. 7a shows the flow released upstream by the gate (control action). Fig. $7 \mathrm{~b}$ presents the evolution of the parameters of the Hayami model expressed as in (5) that clearly shows that they vary with the operating point. Although both LTI $H_{\infty}$ PID and LPV PID satisfy the given control specifications, Table V shows that the performance measured in terms of mean square tracking error achieved by the LPV PID is better than the one provided by the LTI $H_{\infty}$ PID for the whole admissible operating range.

To see more clearly the benefits of using a Smith Predictor, Fig. 8a and 8b present the time response using the proposed LPV PID when the Smith Predictor is used or not. In the case the Smith predictor is not used, the PID controllers are not the same but they have been redesigned considering the delay in the uncertainty weight. It can be seen that the use of the Smith Predictor produces a response with less overshoot. Similar results can also be obtained even with the LTI $H_{\infty}$ PID controller when using or not the Smith Predictor (see Fig. 9a and 9b).

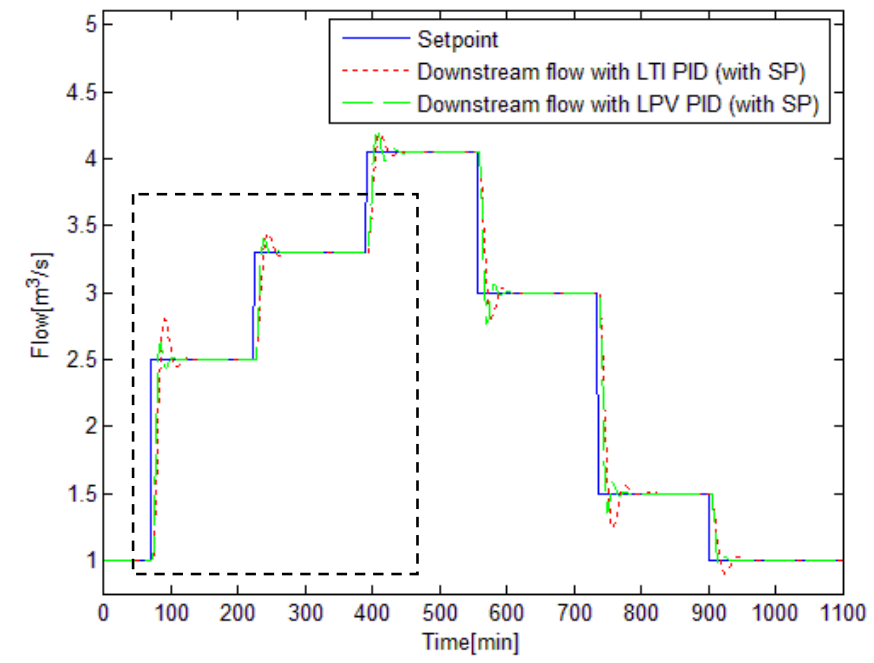

(a)

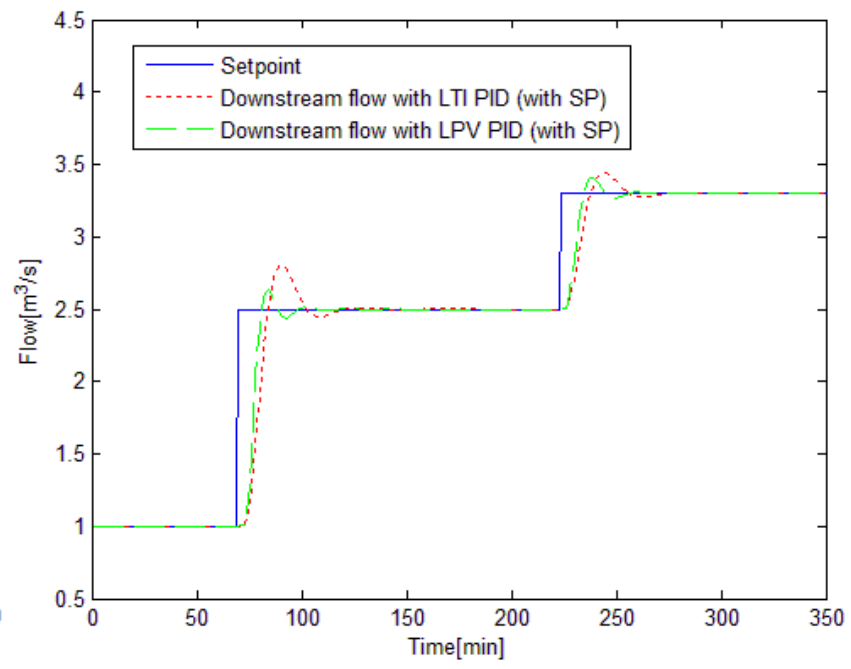

(b)

Fig. 6. (a) Closed-loop response for different operating points, using LPV and LTI control with SP. (b) Detail of the part of the closedloop responses 


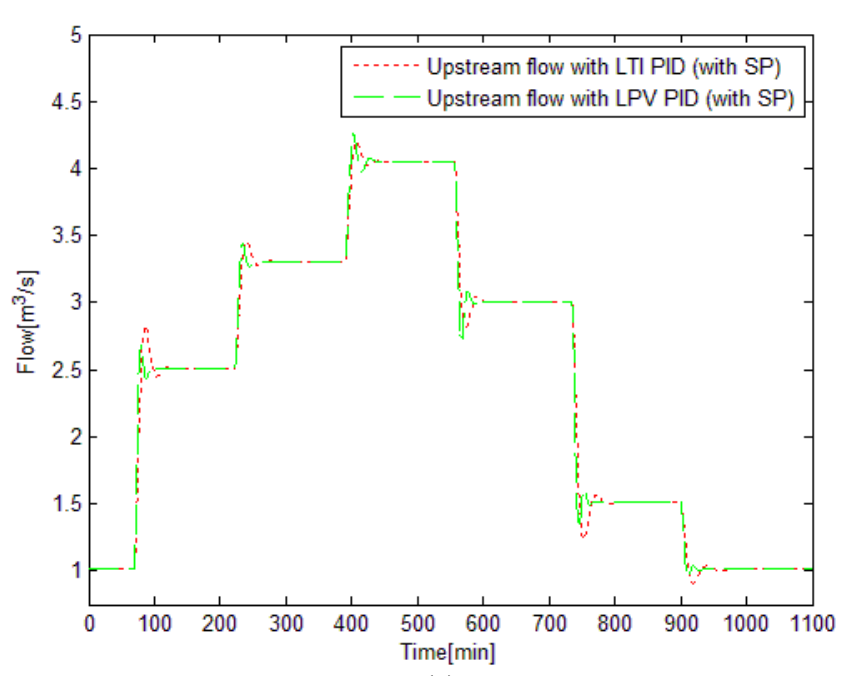

(a)

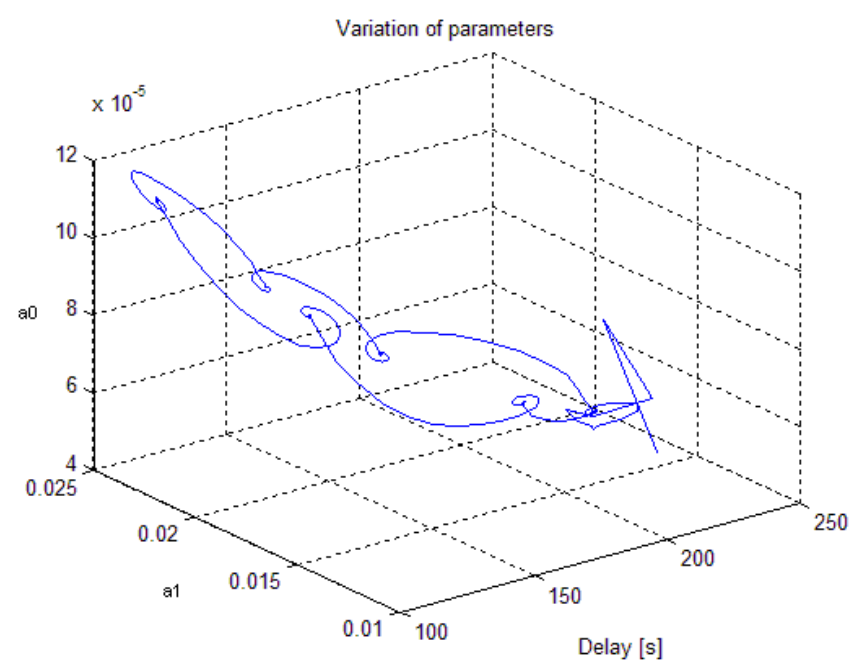

(b)

Fig. 7. (a) Control actions (released upstream flow) corresponding to the operating point changes presented in Fig.6a.

(b) Evolution of the model parameters corresponding to the operating point changes presented in Fig.6a.

Table V: Tracking performance comparison using the LPV and LTI controllers for the operating points presented in Fig. 6a

\begin{tabular}{|c|c|c|c|}
\hline Operating point & $\begin{array}{c}\text { Mean square tracking } \\
\text { error LTI PID+SP }\end{array}$ & $\begin{array}{c}\text { Mean square tracking } \\
\text { error LPV PID+SP }\end{array}$ & $\begin{array}{c}\text { Improvement of the } \\
\text { LPV controller (\%) }\end{array}$ \\
\hline 1 & 18.6 & 14.4 & 22.4 \\
\hline 2 & 4.73 & 3.78 & 20.1 \\
\hline 3 & 4.63 & 4.05 & 12.5 \\
\hline 4 & 6.88 & 5.56 & 19.2 \\
\hline 5 & 12.48 & 8.88 & 28.8 \\
\hline 6 & 1.61 & 1.13 & 30 \\
\hline
\end{tabular}

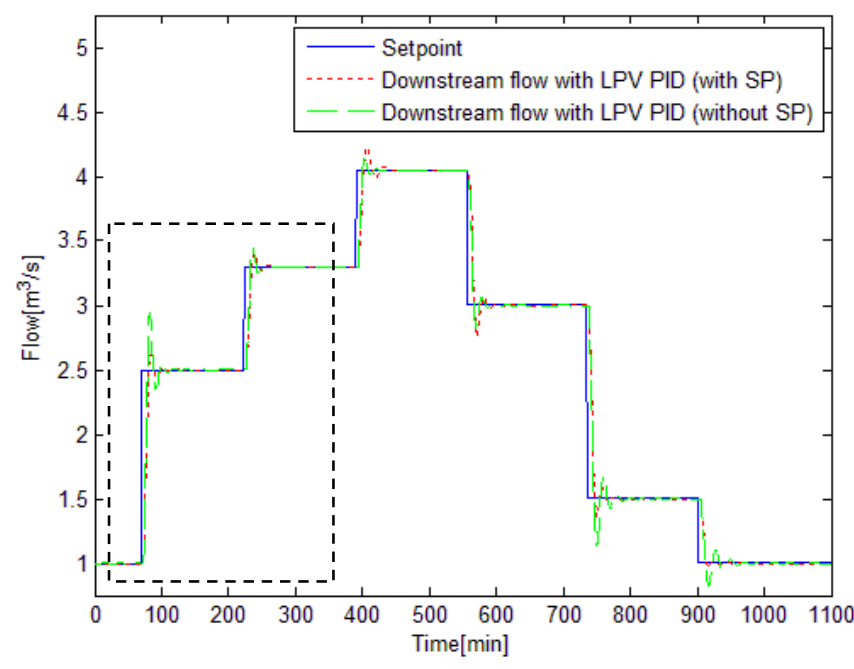

(a)

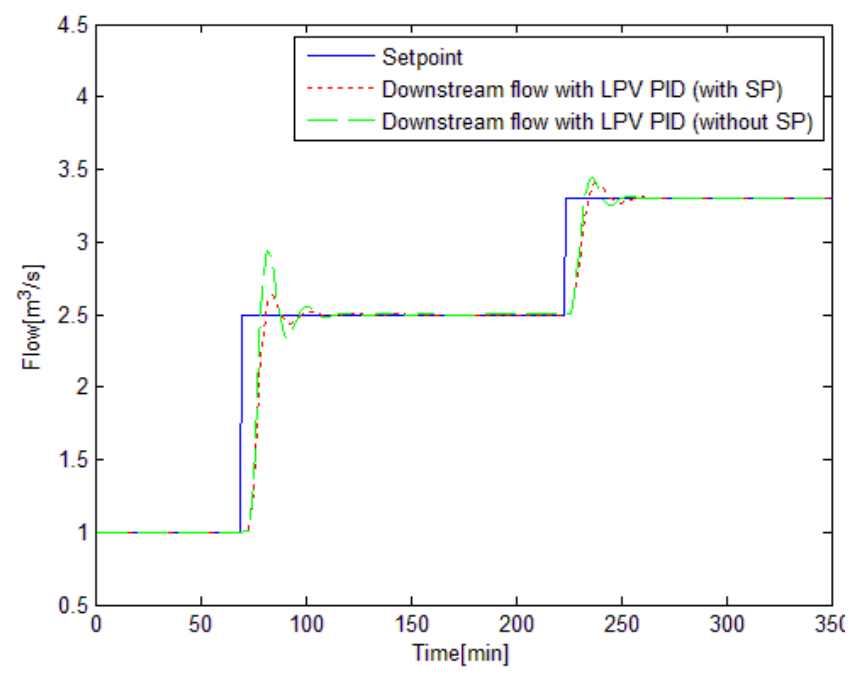

(b)

Fig. 8. (a) Closed-loop response for different operating points using LPV control with and without SP. (b) Detail of the part of the closed-loop responses. 


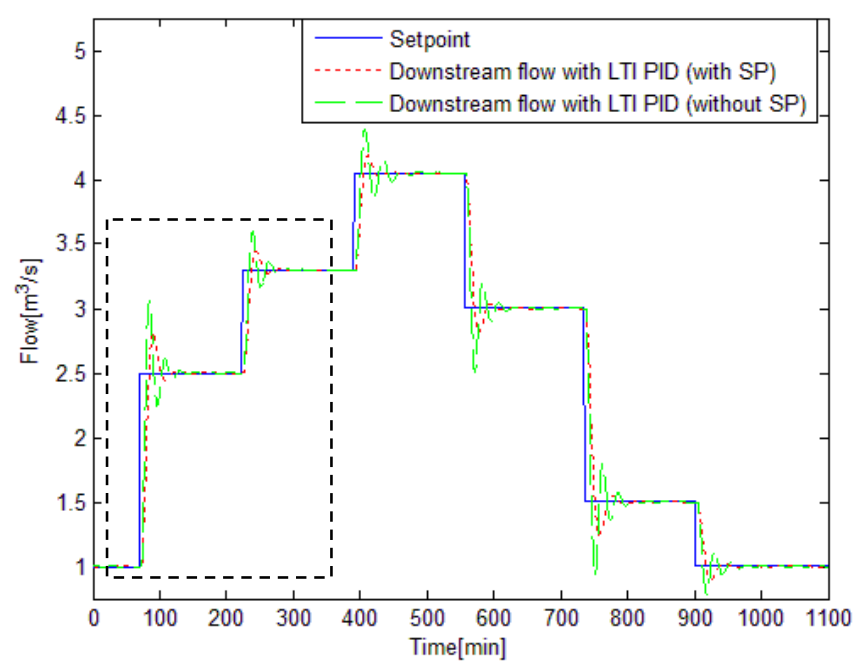

(a)

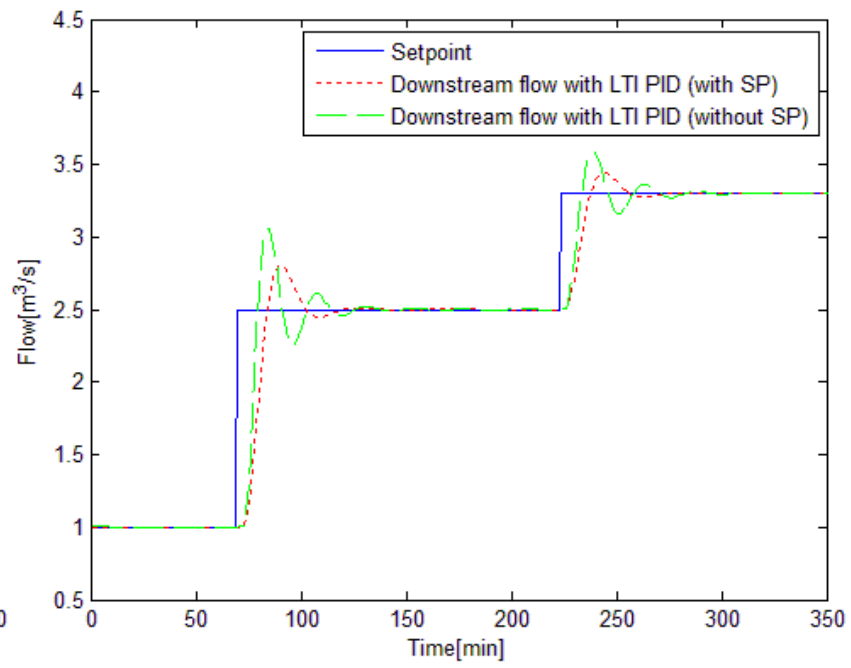

(b)

Fig. 9. (a) Closed-loop response for different operating points using LTI control with and without SP. (b) Detail of the part of the closedloop responses.

\section{CONCLUSIONS}

In this paper, a gain scheduled Smith PID controller has been proposed for the control of an open flow canal system that allows dealing with large variations in operating conditions. An LPV control oriented model for the open-flow channel system based on the Hayami model model has been used. Exploiting the second order structure of this model, an LPV PID controller has been designed using $H_{\infty}$ and pole placement using LMI regions. The controller structure includes a Smith Predictor, real time estimated parameters from measurements (including the known part of the delay) that schedule the controller and predictor and unstructured dynamic uncertainty which covers the unknown portion of the delay. Finally, the proposed controller has been successfully validated in a real case study based on single real reach canal: the Lunax Gallery at Gascogne (France).

\section{REFERENCES}

[1] Litrico, X. \& Fromion, V. (2009). Modelling and control of hydrosystems, London: Springer-Verlag.

[2] Litrico, X. \& Georges, D. (1999a). Robust continuos-time and discrete-time flow control of a dam-river system: (I) Modelling. Appl. Mathematical Modelling, 23(11), pp.809-827.

[3] Gómez, M., J. Rodellar, J., \& Mantecon, J.A. (2002). Predictive control method for decentralized operation of irrigation canals. Applied Mathematical Modelling, 26, pp. 1039-1056.

[4] Schuurmans, J., Clemmens, A.J., Dijkstra, S., Hof, A. \& Brouwer, R. (1999). Modeling of irrigation and drainage for controller design. Journal of irrigation and drainage engineering, Nov/Dec., pp.338-343.

[5] Litrico, X. \& Fromion, V. (2004). Simplified modeling of irrigation canals for controller design. Journal of Irrigation and Drainage Engineering, 130 (5), pp.373-383.

[6] Weyer, E. (2001). System identification of an open canal. Control Engineering Practice, 9, pp. 1289-1299.

[7] Ooi, S.K., M. P. M. Krutzen, and E. Weyer (2005). On physical and data driven modelling of irrigation channels. Control Engineering Practice, 13 (4), pp. 461-471 
[8] Litrico, X. \& Fromion, V. (2006), Hळ Control of an Irrigation Canal Pool with a Mixed Control Politics. IEEE Transactions on Constrol Systems Technology, 14(1), pp.99-111.

[9] Feliu-Batlle, V., Rivas Pérez, R. \& Sánchez Rodríguez, L. (2007). Fractional Robust Control of Main Irrigation Canals with Variable Dynamic Parameters. Control Engineering Practice, 15(6), 673-686.

[10] Rabbani, T.S., Di Meglio, F., Litrico, X. \& Bayen, A.M. (2010). Feed-Forward Control of Open Flow Using Differential Flatness. IEEE Transactions on Control Systems Technology, 18(1), pp. 213-221.

[11] Rugh, W. \& Shamma, J. (2000). Research on gain scheduling. Automatica, 36, pp. 1401-1425.

[12] Belforte, G., Dabbene, F. \& Gay, P. (2005,), LPV Approximation of Distributed Parameter Systems in Environmental Modeling. Environmental Modelling and Software 20(8), pp. 1063-1070.

[13] Puig, V., Quevedo, J, Escobet, T., Charbonnaud, P., Duviella, E. (2005). Identification and Control of an Open-flow Canal using LPV Models. In 44th IEEE Conference on Decision and Control \& 8th European Control Conference (CDC-ECC'05), Seville, Spain.

[14] Litrico, X. \& Georges, D. (1999b). Robust continuous-time and discrete-time flow control of a dam-river system. (II) Controller design. Appl. Mathematical Modelling, 23(11), pp.829-846.

[15] Shamma J.S. \& Athans, M. (1990). Analysis of gain scheduled control for non linear plants. IEEE Trans. Automatic Control, 35(8), pp.898-907.

[16] Apkarian, P. \& Gahinet, P. (1995a). A convex characterization of gain-scheduled $\mathrm{H}_{\infty}$ controllers. IEEE Trans. on Automatic Control, 40, pp. 853864.

[17] Apkarian, P., Gahinet, P., \& Becker, G. (1995b). Self-Scheduled $\mathrm{H}_{\infty}$ Control of Linear Parameter-Varying Systems: A Design Example. Automatica, 31(9), pp. 1251-1261.

[18] Tanaka, K \& Wang H.O. (2002). Fuzzy control systems design and analysis. A linear matrix inequality approach. John Wiley \& Sons.

[19] Leith, D.J., Shorten, R.N. \& Leithead, W.E. (2003). Issues in the design of switched linear control systems: A benchmark study. International Journal Adaptive Control Signal Process, 17, pp.103-118.

[20] Mattei, M. (2003). Robust multivariable PID control for linear parameter varying systems. Automatica, 37, 2003.

[21] Bolea, Y. \& Blesa, J. (2000). Open-flow canal simulator (ICS). Automatic Control Dept., Technical Univ. of Catalonia, Internal Report.

[22] Blesa, J., Puig V., Bolea, Y. Fault detection using interval LPV models in an Open-flow Canal. Control Engineering Practice, 18(5): 460-470.

[23] Duviella, E., Puig, V., Charbonnaud, P., Escobet, T., Carrillo, F. J. \& Quevedo, J. (2010). Supervised Gain-Scheduling Multi-Model vs LPV IMC of Open-Channel Systems for Large Operating Conditions. Journal of Irrigation and Drainage Engineering (in press).

[24] Skogestad, S. \& Postlethwaite, I. (1997). Multivariable feedback control. Analysis and Design. John Wiley \& Sons.

[25] Sánchez-Peña, R.S. \& Sznaier, M. (1998). Robust Systems Theory and Application. John Wiley \& Sons.

[26] Ge, M., Chiu, M.-S. \& Wang, Q.-G. (2002). Robust PID controller design via LMI approach. Journal of Process Control, 12, pp. 3-13.

[27] Chilali, M., Gahinet, P. \& Apkarian, P. (1999). Robust Pole Placement in LMI Regions. IEEE Trans. Automatic Control, 44(12), pp. $2257-2270$.

[28] Kristiansson, B. ; Lennartson, B. (2006 ). Robust tuning of PI and PID controllers: using derivative action despite sensor noise. IEEE Control Systems Magazine. 26 (1) pp. 55-69

[29] Normey-Rico, J.E., Camacho, E.F. (2007) Control of Dead-time Processes. Springer.

[30] Astrom, K, Hägglund, T. (1995) PID Controllers: Theory, Design and Tuning. International Society for Measurement and Control.

[31] Goodwin, G., Graebe, S., Salgado, M. (2001). Control System Design. Prentice Hall. 